msh-mss Mathématiques et sciences humaines

198 | Eté 2012

Varia

\title{
Les exemples de la Russie et de la Rome Antique
}

Log-periodic expansions of Russian and Roman Territories

Ivan Brissaud, Jean Chaline, Pierre Grou et Laurent Notalle

\section{(2) OpenEdition}

Journals

Édition électronique

URL : http://journals.openedition.org/msh/12241

DOI : $10.4000 / \mathrm{msh} .12241$

ISSN : 1950-6821

Éditeur

Centre d'analyse et de mathématique sociales de l'EHESS

Édition imprimée

Date de publication : 12 juillet 2012

Pagination : $29-48$

ISSN : 0987-6936

\section{Référence électronique}

Ivan Brissaud, Jean Chaline, Pierre Grou et Laurent Notalle, « Les exemples de la Russie et de la Rome Antique ", Mathématiques et sciences humaines [En ligne], 198 | Eté 2012, document 1460, mis en ligne le 13 juillet 2012, consulté le 23 juillet 2020. URL : http://journals.openedition.org/msh/12241 ; DOI : https://doi.org/10.4000/msh.12241 


\title{
EXPANSIONS TERRITORIALES LOG-PÉRIODIQUES. LES EXEMPLES DE LA RUSSIE ET DE LA ROME ANTIQUE
}

\author{
Ivan BRISSAUD1, Jean CHALINE², Pierre GROU³ ${ }^{3}$ Laurent NOTTALE ${ }^{4}$
}

\begin{abstract}
RÉSUMÉ - Nous nous proposons d'étudier la chronologie des expansions de l'Empire Russe et de la Rome Antique. Ces expansions se sont déroulées durant plusieurs siècles dans des contrées fort différentes, à des époques également différentes, avant de s'achever par des crises majeures qui ont débouchées dans le premier cas sur la révolution bolchevique de 1917 et dans l'autre par son implosion laissant à Byzance la proéminence parmi les pays méditerranéens. L'histoire de ces conquêtes et l'étude des deux chronologies devraient permettre de caractériser ces extensions territoriales et, en particulier, de montrer que ces évolutions ont un comportement log-périodique déjà mis en évidence dans un grand nombre d'exemples ne concernant pas uniquement les Sciences Sociales et Humaines.

MOTS CLÉS - Évolution, Expansions russes, Expansions romaines, Log-Périodicité, Universalité

ABSTRACT - Log-periodic expansions of Russian and Roman Territories

Territories of the Russian Empire and of the Roman Empire expanded over a period of several centuries, but independently of the time and of the geographic areas. Their evolution took the form of a series of jumps followed by a pause indicating a deceleration mode from a critical time. Such a system could be described by a log-periodic law. This type of behaviour can observed in the chronology of various phenomena (species evolution of echinoderms, dinosaurs, rodents, equids, primates and hominids; human ontogeny, financial crashes and earthquakes...)..
\end{abstract}

KEYWORDS - Evolution, Log-Periodicity, Roman territories, Russian territories, Universality

\section{INTRODUCTION}

Un certain nombre de phénomènes physiques présente un caractère non-linéaire avec des évolutions discontinues marquées par des sauts discrets, liés dans certains cas à des structures fractales. Dans le domaine des sciences de la Terre, les séismes sont, parmi d'autres, un exemple classique avec la succession des précurseurs et des répliques qui

1 CNRS/IN2P3-Université Paris-Sud, 245 chemin du Camp de Lambert, 13400 Aubagne brissaud.ivan@orange.fr

2 Laboratoire de Bio-géosciences (UMR CNRS 5561), Université de Bourgogne, 6 boulevard Gabriel, 21000 Dijon jean.chaline@orange.fr

3 Centre d'études sur la mondialisation, les conflits, les territoires et les vulnérabilités (CEMOTEV), UFR de sciences sociales, Université de Versailles-Saint-Quentin-en-Yvelines, 78280 Guyancourt, piergrou@noos.fr

4 Laboratoire Univers et Théories (LUTH) (UMR8102 CNRS), Université Paris 7, Observatoire ParisMeudon,92190 Meudon, laurent.nottale@obspm.fr 
précèdent et suivent un séisme majeur. Il a été montré, en particulier par D. Sornette et C.G. Sammis [1995], D. Sornette, A. Johansen, B. Bouchaud [1996], A. Johansen et D. Sornette [2001], D. Sornette [2002], que ces événements obéissent à des lois logpériodiques caractérisées par des dimensions fractales complexes et pouvaient dans certains cas être identifiés à des phénomènes critiques. Comme le montre l'exemple des séismes, ces évolutions présentent des accélérations et des décélérations vers ou à partir d'un temps critique Tc, lors d'un déroulement n'obéissant qu'à l'écart de temps avec Tc.

Une justification de la nature des résultats précédents a été proposée dans le cadre de la Théorie de la Relativité d'Échelle [Nottale, 1993]. Dans cette théorie (TRE), on étend le principe de Relativité aux changements d'échelle, ce qui unifie, dans le cadre d'un espace-temps fractal, les lois quantiques et classiques. L'origine des structures logpériodiques fractales des arbres d'évolution y trouve une explication naturelle. En utilisant ces outils, un grand nombre d'analyses d'évolutions log-périodiques dans plusieurs domaines des sciences physiques et de la vie, des sciences sociales et humaines ont été publiées [Chaline, Nottale et Grou, 1999; Nottale, Chaline et Grou 2000(a), 2009 ; Grou, Nottale et Chaline, 2002]. Confirmant les succès de l'application de la TRE, d'autres exemples sont venus s'ajouter récemment à ces études [Brissaud, 2007, 2009(a, b)].

Dans ce présent travail nous voulons montrer l'intérêt d'appliquer la TRE au domaine des sciences historiques en examinant l'évolution territoriale des grands Empires russe et romain sur plusieurs siècles et dans leur diversité pour vérifier si eux aussi obéissent à des lois log-périodiques.

Rappelons qu'un système log-périodique évolue de saut en saut survenant aux temps Tn, vers un temps critique Tc. Comme le nom l'indique, il y a une périodicité des quantités $\log [\mathrm{Tn}-\mathrm{Tc}]$. Dans l'exemple envisagé ici nous allons vérifier si les deux empires cités plus haut évoluent par grandes étapes qui obéissent à des relations déduites de la TRE. Celle-ci montre qu'une évolution s'effectue en suivant les relations suivantes reliant les $\mathrm{Tn}$ :

$$
T n+1=[(g-1) T c+T n] / g
$$

avec

$$
g=(T n-T n-1) /(T n+1-T n) \text { et } T c=[g T n+1-T n] /(g-1)
$$

Tc étant respectivement le temps critique et $g$ le coefficient d'autosimilarité qui donne des informations sur le rythme de cette évolution. Le lecteur peut retrouver l'origine de ces relations dans les références [Nottale, Chaline et Grou, 2000(b), 2009]

\section{EXPANSIONS TERRITORIALES DE LA RUSSIE DU XIVe AU XXe SIÈCLE}

Comme l'a souligné J. Radvanyi [2004], la Russie a eu, depuis la fin de la «Russie kiévienne » au XIIe siècle, une expansion tout à fait particulière, même unique par sa durée de cinq à six siècles environ, qui lui a fait traverser diverses organisations sociales, par son histoire souvent violente, par son étendue exceptionnelle et inégalée (environ 22 millions de kilomètres carrés), par sa diversité en populations et ressources, par ses climats extrêmes, etc. Également il faut souligner que l'extension de la Russie s'est opérée dans plusieurs directions (Europe occidentale évidemment, mais très largement vers la Sibérie et l'Arctique, le Caucase et l'Asie centrale). Cette évolution a son origine, tout au moins au départ, dans la recherche d'encrages (mers, fleuves...) pour se protéger des agressions continuelles et venues de toutes parts, dans la recherche de débouchés maritimes et fluviaux pour son commerce et finalement dans l'exploitation de richesses naturelles. 


\subsection{SURFACES CUMULÉES DE LA RUSSIE}

Nous avons pu établir la chronologie de l'expansion russe jusqu'à l'époque soviétique à l'aide de plusieurs sources, plus particulièrement de l'Atlas historique de J. Channon [1997]. Quant aux ouvrages référencés, ils ont servi à dater de nombreuses étapes de ce développement. Divers ouvrages et encyclopédies (y compris sur Internet) ont permis de confirmer certaines dates et les étendues de nombreuses annexions. La figure 1 donne la surface cumulée en fonction du temps, entre le XIVe et XXe siècle. Elle montre très nettement la succession, jusqu'au XXe siècle, de 3 périodes d'extensions, chacune suivie de pause.

Nous avons vérifié dans l'étude sur le développement de l'Empire romain (cf.plus loin) que chaque poussée de conquêtes obéit à une fonction logistique d'une forme classique :

$$
S(t)=S m /\left(1+\exp -\left(\left(t-t^{\circ}\right) / D t\right)\right.
$$

où $S m$ est la valeur maximum atteinte au temps $t^{\circ}$ et $D t$ le temps de montée de $S(t)$ pour passer des valeurs $S m \times 0,1$ à $S m \times 0,9$.

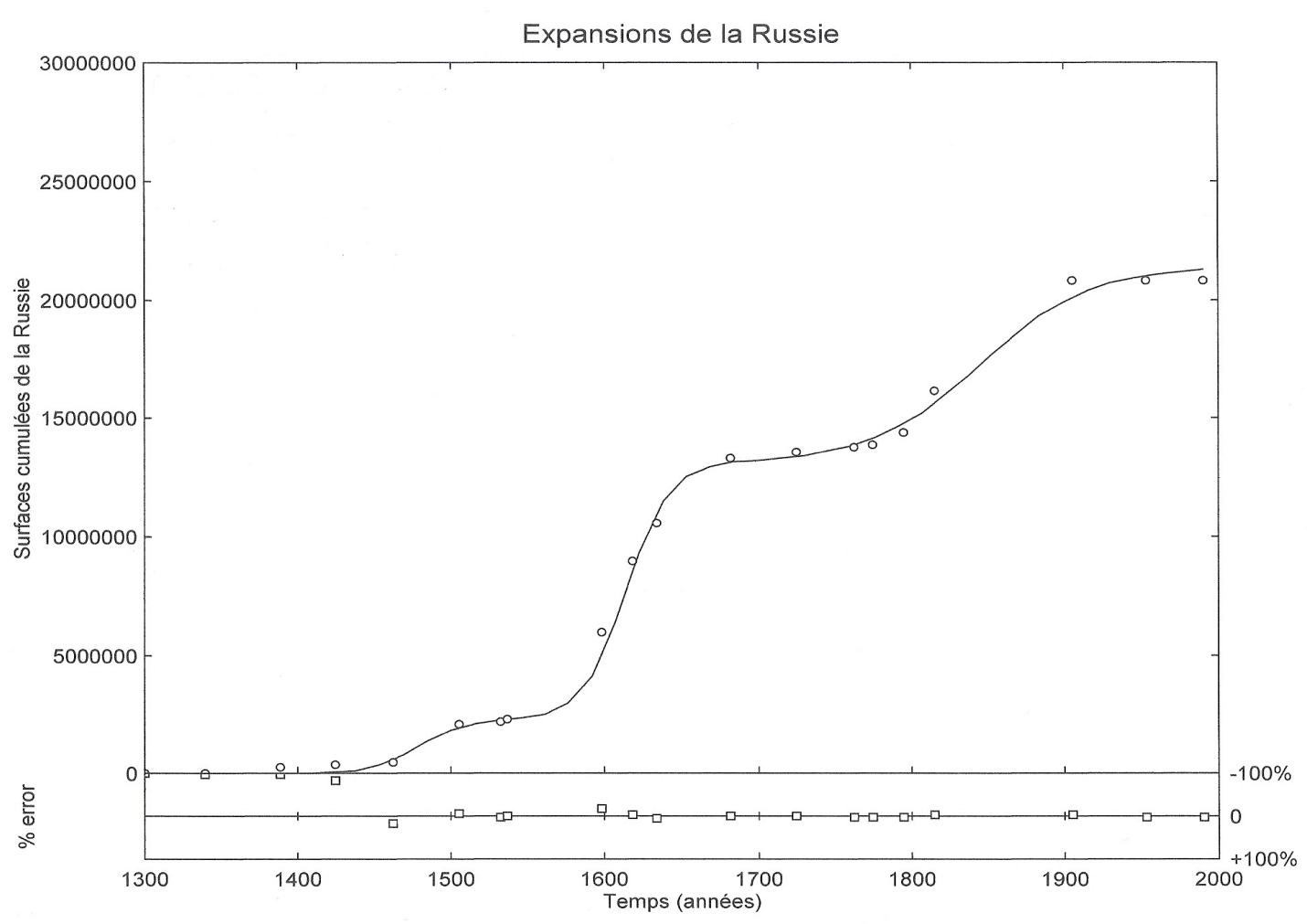

FIGURE 1. Surfaces cumulées de la Russie du XIVe au XXe siècle.

Le Tableau 1 présente les paramètres des 3 fonctions logistiques donnant la courbe théorique qui s'ajuste parfaitement sur les données

En utilisant le logiciel «Loglet Lab» nous pouvons ajuster 3 logistiques sur les données. La Figure 1 présente la courbe lissant au mieux ces données après une recherche par moindres carrés. L'accord est très satisfaisant. Chacune de ces logistiques est présentée sur la Figure 2 ; les paramètres correspondants sont donnés sur le Tableau 1. 


$\begin{array}{cccc}S m & 1910000 & 11400000 & 9900000 \\ t^{\circ} & 1472 & 1607 & 1858 \\ D t & 44 & 97 & 160\end{array}$

TABLEAU 1. Paramètres des 3 logistiques lissant les données de la Figure 1

Annexions de la Russie

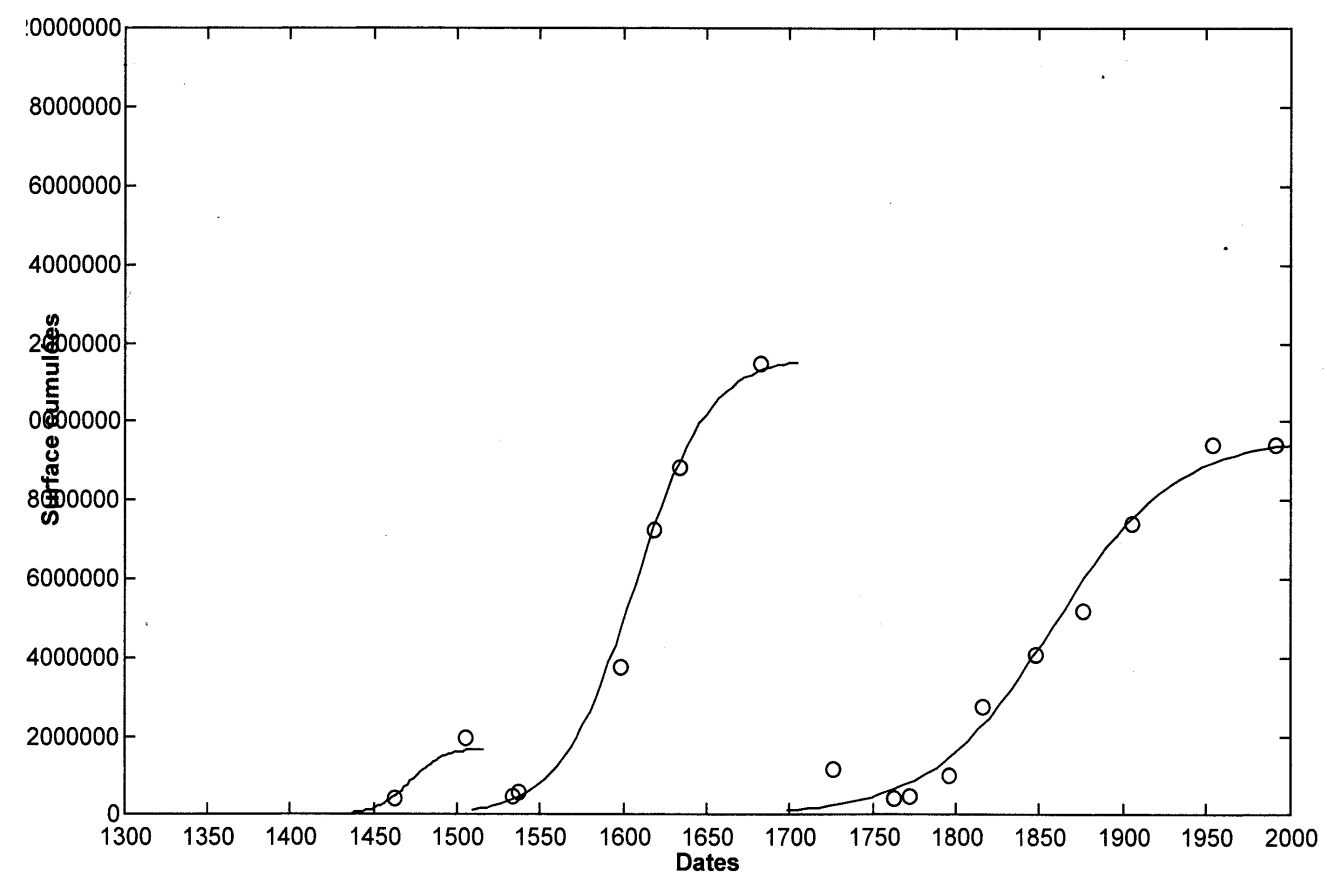

FIGURE 2. Décomposition de la courbe de la Figure 1 en trois fonctions logistiques

À partir des trois dates $t^{\circ}$ définies pour ces logistiques (cf. Figure 2) nous proposons une évolution log-périodique des extensions russes. En utilisant la formule (I) on obtient les paramètres suivants :

$$
\begin{array}{ll}
\text { Temps critique } & T c: 1290+/-20 \\
\text { Facteur d'autosimilarité } & g: 1,71+/-0,04
\end{array}
$$

Pour justifier la validité de l'hypothèse log-périodique nous pouvons remarquer :

- la valeur de $g$ obtenue correspond à une des familles de coefficients d'autosimilarité déjà déduites de diverses analyses : des pics de probabilité sont obtenus de manière systématique autour de $g=3 k / 4$ pour $k=1(31 / 4=1.316), k=2(31 / 2=1.732)$ et $k=3(33 / 4=2.280)$ [Nottale, 2010] ;

- la partie centrale de l'actuelle Russie était occupée par un grand nombre de petites principautés sous domination des Tatars. Le temps critique Tc correspond au moment où, à la fin du XIIIe siècle, Daniel (mort en 1304), devenu Grand-Prince de Moscou, commença en grignotant les principautés voisines, à étendre la surface de la Moscovie alors que celle-ci n'en était qu'une parmi d'autres ;

- À l'aide de la formule (I) il est possible de calculer les dates des annexions qui, après le temps critique, précédent le XVe siècle :

$$
\begin{aligned}
& T 1=1354+/-20 \\
& T 2=1400+/-20
\end{aligned}
$$


Or, en cherchant à décomposer avec le code Loglet Lab la partie de la courbe comprise entre 1300 et 1500 (cf. Figure 1), on met en évidence une fonction logistique centrée sur l'an 1388, ayant un temps de montée $D t=139$, pour une saturation $S m=540000$. Ce résultat est parfaitement cohérent avec la date calculée T2. Ces chiffres confirment qu'à la fin du XIVe siècle la surface de la Moscovie était supérieure d'un ordre de grandeur à celle qu'elle avait eu cent ans auparavant. Par contre, faute de données, il est difficile de confirmer avec certitude la présence d'une fonction plus ancienne, vers 1350, plusieurs vagues de conquêtes étant trop proches pour être différentiées.

\section{2 ÉVOLUTION HISTORIQUE DES ANNEXIONS}

Les extensions du territoire russe se sont faites par vagues comme le montre la Figure 3. Chaque vague s'étend sur un intervalle de temps de 100 à 200 ans environ. En considérant (en la globalisant !) l'histoire de la Russie il est possible de caractériser assez schématiquement chacune des quatre vagues qui ont été identifiées plus haut :

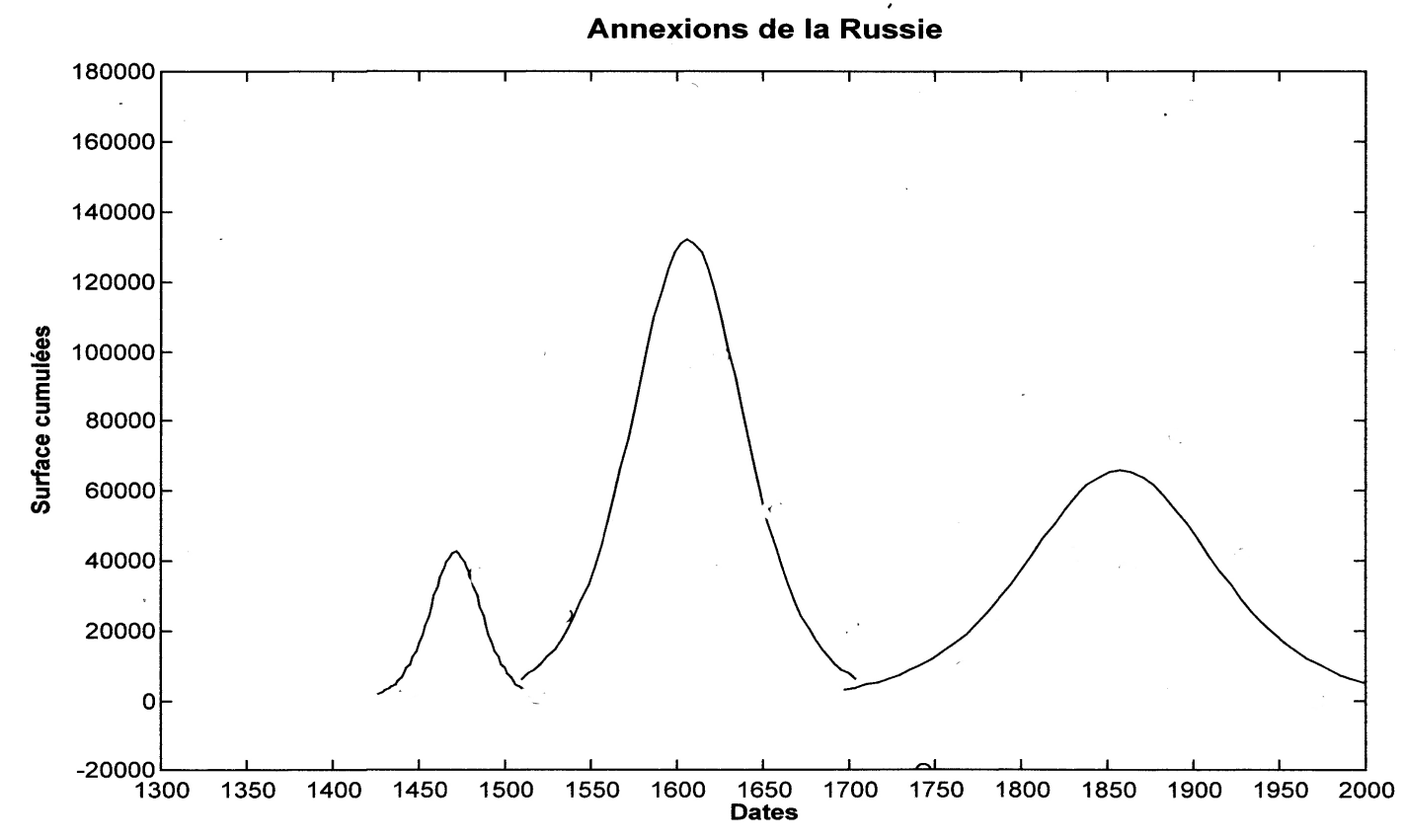

FIGURE 3. Fonctions dérivées des 3 fonctions logistiques

a) Au XIIIe siècle la région centrale de l'actuelle fédération de Russie était occupée par une mosaïque de principautés sous la domination tatare (Mongole), domination qui devait durer plus de deux siècles. Outre le fort tribut qu'elles versaient aux Tatars, ces principautés étaient gouvernées par des princes choisis par les Tatars, princes qui suivaient la politique définie par eux ! Elles subissaient également les attaques des Baltes, des Suédois, des Chevaliers Teutoniques (ou Porte-Glaive)... Ces deux derniers furent vaincus par le fameux Alexandre dit «Nevski». Sous l'impulsion des princes successifs de la Moscovie débute à la fin de ce siècle la longue lutte contre les Tatars ${ }^{5}$

5 La période 1223-1480 nommée «Le temps des ténèbres » fut particulièrement difficile : A. Préchac [2001] indique que durant cet intervalle de temps, on compte 28 années de famine générale, 23 épidémies de peste, 90 guerres intérieures, 160 guerres extérieures avec les Mongols, les Lituaniens, les Germaniques, les Suédois, les Bulgares, les Hongrois, etc. 
avec la première grande défaite de ceux-ci à Koulikovo sur le Don (1380) par l'armée du Grand-Prince Dimitri Ivanovitch «Donskoï». Cette victoire russe intervient après les succès du prince Daniel (fils d'Alexandre Nevski) qui fixa sa résidence à Moscou (1263), alors une petite ville, pour des raisons stratégiques (carrefour de voies fluviales et non terrestres trop dangereuses). Il y installa la dynastie des Danilovitch et fut le premier à accroître le territoire de la Moscovie qui commença à dominer les principautés voisines. Le métropolite se fixa également à Moscou à la même époque ce qui renforça l'influence de la Moscovie. La Figure 4 donne la carte des expansions de la Russie à cinq moments historiques depuis la Moscovie de 1300 jusqu'à l'an 1905.

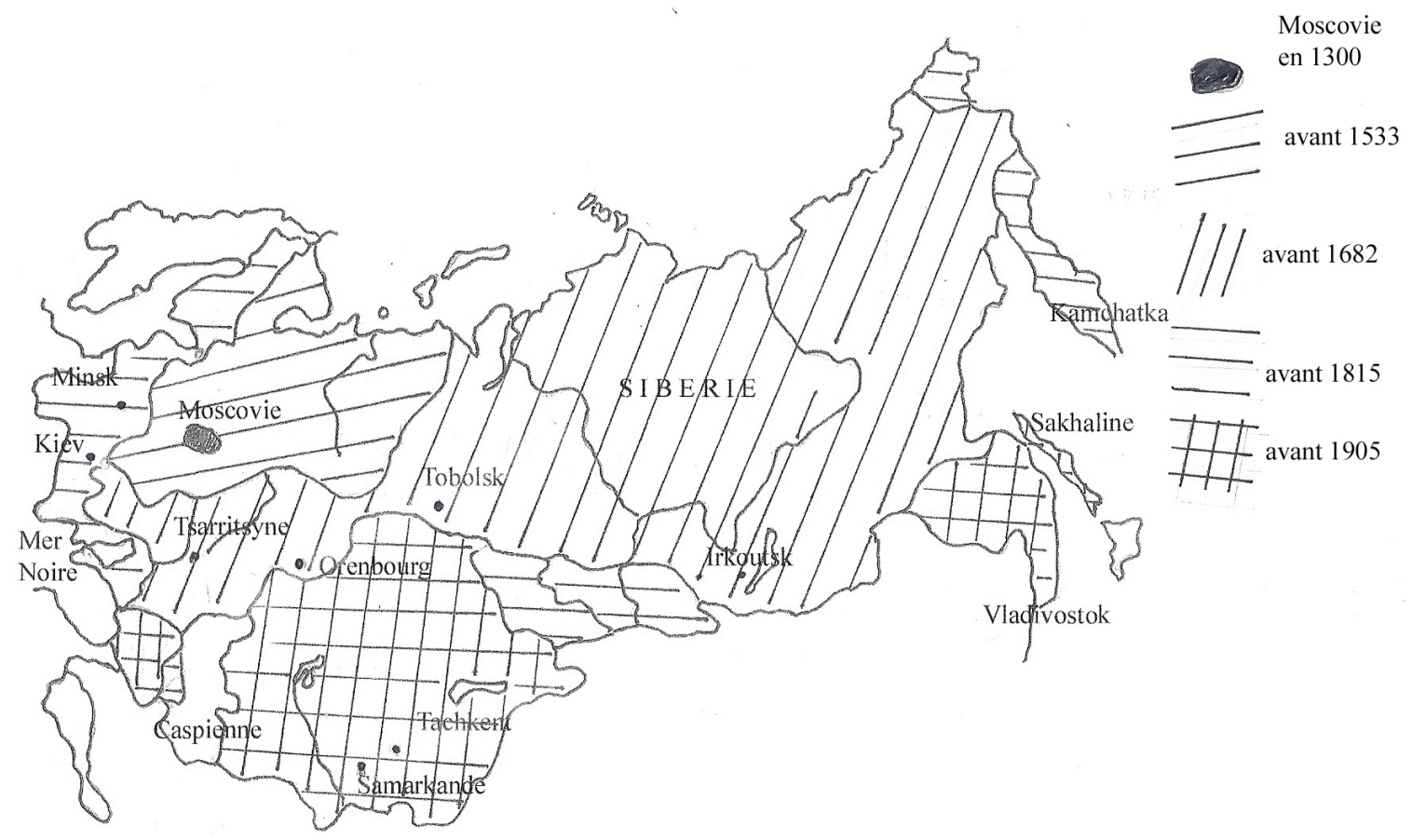

FIGURE 4. Carte des expansions successives de la Russie à cinq moments historiques depuis 1300 jusqu'à 1905

b) Au cours du XVe siècle se développe la lutte entre les princes féodaux et les partisans de l'unification des terres russes parmi lesquels les membres d'une nouvelle classe sociale qui se forme, la noblesse. Celle-ci, soutenue par le clergé orthodoxe dont les biens étaient convoités par les féodaux, adhère au projet d'un Etat fort pour consolider sa position. Dans sa lutte contre la haute aristocratie Ivan III, dit « le Grand », (14621505), le premier autocrate, consolida en 1497 par une nouvelle réglementation la centralisation de la justice et de l'administration et légalisa le servage sur tout le pays. Ces mesures lui donnèrent différents moyens d'absorber diverses petites principautés de sorte que la Moscovie atteignit une surface considérable, le but recherché étant toujours de se protéger des agressions qu'aucun obstacle naturel ne pouvait contenir. Un des résultats fut de donner un lustre supplémentaire à la Moscovie dans plusieurs domaines et spécialement en peinture (par exemple les icones d'Andreï Roublev) et en architecture (édification des églises du Kremlin de Moscou), etc.

c) Le XVIe siècle voit s'affirmer le pouvoir central face aux princes et boyards qui conservent une grande influence locale. Ivan IV dit «Le Terrible» (1547-1584) se proclame Tsar (César) et engage une lutte sans pitié contre ces derniers qui avaient tenté de le mettre à l'écart. Ce renforcement du pouvoir donna les moyens d'une politique 
extérieure énergique ; il en résulta l'écrasement définitif des Tatars. Si l'expansion vers l'Occident fut bloquée, le grand projet demeure, avec un large succès, le début de la « colonisation» des territoires peu peuplés de la Sibérie Occidentale. Ceci se fit tout d'abord par l'intermédiaire de riches commerçants (les Stroganov en particulier) qui levèrent une armée privée formée de Cosaques. Là, le but de ces actions fut principalement le commerce fort lucratif des fourrures. Au long de cette période faste on note de grands progrès dans l'agriculture, dans l'élevage et le commerce. Pour mener à bien ces activités, spécialement l'introduction et le développement de l'agriculture dans des régions annexées, le servage fut étendu de plus belle dans un cadre féodal toujours plus marqué. Le début du siècle suivant fut marqué par des difficultés internes (assassinats, faux tsars...) au cours d'une période nommée le «temps des troubles » qui s'achève avec l'avènement des Romanov en 1613. Le rythme des annexions s'en trouva fortement ralenti.

d) Conscient de l'état arriéré de la société russe, Pierre Le Grand (1682-1725) adopta une politique d'ouverture vers l'Europe qu'il visita deux fois et, comme ses prédécesseurs, rechercha des débouchés sur la mer. Après une longue suite de combats, il absorba divers pays bordant le golfe de Botnie. Une des conséquences fut la création de Saint-Pétersbourg (1703) et le transfert dans cette nouvelle ville de la capitale de la Russie. Simultanément il entreprit une réorganisation du pays, créa des entreprises minières et industrielles civiles et militaires (dans l'Oural métallifère), des établissements d'enseignement, aménagea des voies fluviales, élimina la menace suédoise (Poltava 1709)... tout en renforçant, pour imposer ces réformes, son pouvoir absolu. Enfin, il proclama «L'Empire Russe ». Après des années mouvementées sous le règne de plusieurs impératrices, la Grande Catherine (1762-1796) poursuivit la même politique. Elle put annexer plusieurs régions dont la Crimée, une partie de la Biélorussie. Au XIXe siècle, après le partage de la Pologne (1772-95), les guerres ne cessent pas : contre la France, la Turquie d'où la guerre de Crimée menée par l'Angleterre et la France en 1853-55. Mais, s'il y a des échecs de ce côté-là, les conquêtes continuent dans le Caucase : après la Géorgie (1813), l'Arménie et l'Ossétie (1828/30), la Tchétchénie et le Daghestan (1859) et l'Arda khan (1878). En Asie Centrale le grignotage se poursuit : le Kazakhstan (1847), l'Ouzbékistan (1855), Boukhara et le Tadjikistan (1868), le Khiva et le Turkménistan (1873) et enfin le Pamir (1895). Mais à l'intérieur de la Russie il y a trop de contradictions entre les besoins et les aspirations de la population et le pouvoir tsariste d'autant que l'influence des «Lumières », les idées de la Révolution Française (connues notamment par les officiers des armées françaises d'occupation en 1814) ont pénétré les milieux intellectuels et cela, alors que se constitue une classe ouvrière formée d'anciens serfs contraints au travail industriel. Après le complot et l'exécution ou la déportation des Décembristes, les grèves, les actions révolutionnaires violentes (attentats après 1870, assassinat du tsar Alexandre II en $1881 \ldots$ ) se poursuivent malgré le renforcement de la répression. Cela annonce le soulèvement de 1905 «le dimanche rouge », à Saint Petersbourg et la mutinerie des marins du Potemkine, preuve de la désagrégation des forces armées avant que le tsar Nicolas II n'engage son pays dans la première Guerre mondiale : les défaites de ses armées provoquent les deux révolutions de février et octobre 1917 qui jetèrent à bas l'Empire Russe. Après l'armistice de Brest-Litovsk, la Russie dut se séparer d'une partie de son territoire (l'Ukraine devient momentanément indépendante) et en particulier de ses conquêtes finlandaise, polonaise et balte. En 1945 elle pourra en récupérer une grande partie ainsi que Sakhaline et les Kouriles prises au Japon qui fut vainqueur de la flotte russe en 1905. Si ces échanges ont une grande importance pour leur aspect géopolitique, ils jouent peu sur la comptabilité spatiale. Ultérieurement rien ne change, si ce n'est l'existence du glacis que constituent les pays d'Europe de l'Est 
lors de la guerre froide. Enfin c'est l'éclatement de l'URSS : La Russie doit se séparer de plusieurs régions du sud: sur le versant occidental l'Ukraine, la Biélorussie ainsi qu'au Caucase et en Asie. Mais c'est une autre histoire qui commence.

On constate donc au cours de ces siècles, sur la façade européenne, une succession de guerres et de coalitions qui conduisent à des conquêtes, des abandons et souvent des reconquêtes, mais avec un solde toujours positif. Nous devons noter la parfaite concordance entre ces faits historiques et l'évolution spatiale de la Russie telle qu'elle a été datée sur la Figure 1.

\subsection{DISCUSSION ET CONCLUSION}

Nous venons de survoler la chronologie des annexions qui ont porté la surface de la Moscovie à 22 millions de kilomètres carrés. Cette évolution est exceptionnelle. J. Radvanyi [2004] a rappelé que cela représente, durant trois siècles, un gain de $55000 \mathrm{~km}^{2}$ par an ; or la surface initiale de la Moscovie n'était que d'environ $45000 \mathrm{~km}^{2}$ ! Ceci montre la multiplicité d'échelles et l'accroissement des espaces économiques et sociaux qui se sont succédé durant cet intervalle de temps de quelques siècles [Grou, 1987].

Dans l'historique donné plus haut nous avons noté que les cycles de conquêtes succédaient à des crises économiques, des difficultés intérieures, des guerres... (par exemple, vers 1670, le soulèvement conduit par Stepan Razine précède le renouveau lié à la montée sur le trône de Pierre le Grand et l'écrasement de la révolte de Pougatchev en 1774 renforce le pouvoir autocratique). Les annexions avaient pour but de chercher à se libérer d'un oppresseur, se protéger, augmenter les débouchés commerciaux, avoir accès à de nouvelles richesses pour un pays de plus en plus vaste... (par exemple, les vastes espaces de la Sibérie annexés sont occupés par le transfert d'une population russe (et aussi de prisonniers politiques) qui va y travailler et y développer l'agriculture).

En considérant de plus près la chronologie exposée précédemment et l'immensité du pays, une constatation s'impose : la complexité de la société russe ne pouvait que s'accroître fortement au cours des différentes étapes : au départ une petite principauté malmenée par les Tatars et ses voisins, puis une série d'agrandissements dus principalement à Ivan III qui codifia l'autocratie devant servir de règle pour la gouvernance russe au cours des siècles suivants. Ivan IV confirma le régime autoritaire en se proclamant Tsar, crée la nation russe ${ }^{6}$ (en imposant la langue de Moscou) et occupa une partie de la Sibérie. Pierre le Grand proclama l'Empire, déplaça la capitale vers l'Europe occidentale ; en annexant plusieurs pays du Caucase et de l'Asie centrale la Russie est devenue un pays plus asiatique et arctique qu'européen, ce qu'elle était pleinement au XIII siècle. Après la révolution d'Octobre 1917, l'URSS cherche à cimenter tous ces territoires en créant des fédérations de républiques, en dotant chaque fédération de structures assez identiques tout en maintenant un fort centralisme moscovite.

La gestion économique dut aussi se complexifier : pour l'agriculture, il fallut, au fil du temps, développer et, en la différenciant, l'adapter aux différentes qualités du sol et aux différents types de propriétaires terriens ; il y eut donc les serfs entrepreneurs, les paysans d'État, en attendant la paysannerie libre... L'industrie naissante fut ajustée aux conditions fortes différentes des régions sous Pierre le Grand. À la même époque toute la structure de l'État a été réorganisée dans le but d'occidentaliser la Russie dans

\footnotetext{
6 Pour définir ces deux derniers siècles A. Prechac [2001] peut écrire : « le XIVe siècle était le siècle où naissait un État, la Moscovie. Le XVe sera celui de la naissance d'une Nation, la Russie ».
} 
beaucoup de domaines. Puis devant les troubles sociaux, le servage déjà renforcé dut, encore, être modifié avant d'être supprimé en 1861. Une évolution vers un régime représentatif s'amorça ; les villes devinrent plus autonomes, la justice remodelée... Par ailleurs les communications sont difficiles, les distances énormes (onze fuseaux horaires d'Est en Ouest !), il fallut construire puis développer les routes, les chemins de fer (d'où les «emprunts russes» lancés par les grandes banques occidentales) et, après la deuxième guerre mondiale, les lignes aériennes, alors que le climat, très rude durant plusieurs mois par an et dans presque toutes les régions, complique leur fonctionnement. En permanence il y eut des blocages qui nécessitèrent des remises en cause. Cela impose des structures d'organisation à différents niveaux d'où une bureaucratie très lourde dont on a vu les limites.

En n'oubliant pas de prendre en compte la période encadrant 1388, on remarque que l'écart entre chacune de ces 4 vagues croît avec le temps : il y a décélération. Comme l'a écrit Grou dans son chapitre Des Fleurs pour Schrödinger [Nottale, Chaline, Grou, 2009] : « on se trouve dans le cas d'une décélération, d'un épuisement de plus en plus lent de capacités innovatrices ». D'autre part, le temps de montée des logistiques $(D t)$ croît également exprimant une diminution de l'efficacité des campagnes d'annexions. Par contre l'ampleur des gains de surfaces (la valeur $S m$ ) passe par un maximum vers l'an 1600, avec la conquête de la Sibérie Occidentale par les Cosaques. C'est l'époque la plus expansionniste. Ultérieurement, c'est moins marquant lors du rattachement des régions du Caucase et de l'Asie Centrale ; par la suite, après 1895, peu d'évolution parce que l'expansion se heurte aux impérialismes rivaux ; pratiquement le statut quo ! Enfin, il faut rappeler que la relation (I) a permis de prévoir le temps critique $T c$ et le temps $T 2$ à des moments spécifiques de l'évolution.

De ce qui précède, il apparaît que les caractéristiques de l'évolution des annexions russes sont celles de la succession d'un grand nombre d'échelles, la complexité croissante, la décélération, la recherche d'un plus grand rendement économique, une prédictibilité, caractéristiques d'une évolution log-périodique définies par [Nottale, Chaline, Grou, 2000(b), 2009 ; Grou, Notale, Chaline, 2002] et rappelés plus haut. Notons que cette loi de log-périodicité s'applique sur plusieurs siècles à un pays qui a considérablement changé durant cet intervalle de temps.

À partir des figures précédentes, il est mis en évidence qu'après des gains en territoires se succédant fortement jusqu'au XVIIe siècle, la croissance spatiale de la Russie s'est ralentie jusqu'à l'époque de l'URSS (d'une surface pratiquement étale) ${ }^{7}$. Nous avons vu que la Rome antique, d'une façon voisine, s'était agrandie jusqu'à une surface maximale atteinte au premier siècle de notre ère et que la politique suivie ultérieurement par l'Empire romain s'affaiblissant a été de protéger ces acquis [Grou, 2010]. C'est exactement ce qu'a fait la Russie au cours du XXe siècle. Coincée entre l'Europe Occidentale, la Chine et l'Empire britannique (avant l'indépendance de l'Inde et du Pakistan en 1947) et ayant atteint les cinq mers l'entourant, elle n'a pu s'agrandir davantage dès la fin du XIXe. Autrement dit, pour différentes raisons données plus haut, la conquête de territoires avait été un projet du pouvoir tsariste avant la limite signifiée par la Révolution bolchevique. Après l'arrêt de ce projet russe, l'URSS n'a pas réussi le sien (construire de manière définitive une nouvelle société industrielle). Cela dit, il convient de se demander quelles ont été les classes sociales motrices de l'expansion territoriale observée sur plusieurs siècles. Comme à l'Ouest de l'Europe, il s'est agi d'une

7 Comment faut-il interpréter l'affirmation du philosophe slavophile I. Kireievski : «L'étendue matérielle de l'empire a absorbé pendant des siècles toute l'énergie du peuple russe ; la croissance matérielle a rendu impossible la croissance spirituelle »? 
alliance entre classe marchande et fraction de la féodalité. La réussite a été exceptionnelle, puisque le résultat a été celui de la conquête du vaste espace observé, mais, contrairement à ce qui s'est passé à l'Ouest, la classe marchande n'en a pas profité pour installer un rapport de force définitif en sa faveur lui permettant de devenir dominante et de se transformer en bourgeoisie industrielle. C'est une alliance entre petite bourgeoisie et prolétariat qui a réalisé la révolution industrielle soviétique, dynamique jusqu'à la limite des années 1970.

En conclusion, toutes les remarques précédentes confirment que la succession des annexions territoriales par la Russie suit bien une loi log-périodique. La présence de cette loi a déjà été manifeste dans des domaines fort différents. Nous allons en voir un autre exemple ci-dessous avec l'Empire romain. Un des enjeux des études futures sera donc de comprendre l'origine de cette universalité.

\section{ANNEXIONS TERRITORIALES ET VOIES DE CIRCULATION SOUS LA RÉPUBLIQUE ET L'EMPIRE ROMAIN}

Le but initial de ce travail fut de vérifier si l'inventaire et la chronologie des voies romaines antiques permettait de rendre compte par une évolution log-périodique du déclin de l'Empire Romain. La principale difficulté de cette étude fut que, si les tracés des voies sont parfaitement établis, la date de certaines ne l'est pas. En effet une datation exige des travaux de recherche, en particulier archéologiques, qui ont bien été menés en Italie, en France... mais de façon moins systématique dans d'autres régions méditerranéennes. Dans une première étape, pour contourner cet obstacle, nous étudions la chronologie des annexions qui se sont succédé jusqu'à la possession complète des pays riverains de la Méditerranée. En effet, les historiens ont montré que la construction des routes et les conquêtes s'étaient effectuées de façon liée. Comme souvent, les commerçants sont utilisés pour préparer et réaliser une annexion. Ainsi Chevallier [1997] rappelle dans son ouvrage qu'en Grande-Bretagne des commerçants, qui connaissaient bien la région, jouaient le rôle de guides de l'armée romaine. Mais plus tard, quand les ennemis de Rome ont menacé les frontières, les routes furent nécessaires pour convoyer rapidement des troupes afin de défendre l'empire. On annexait, le cas échéant, d'autres pays voisins pour faciliter ces défenses afin de réduire les lignes de front. Enfin, lors du crépuscule de l'Empire, ce réseau de routes fut emprunté (en sens inverse) par les envahisseurs.

\subsection{LES EXPANSIONS DE LA ROME ANTIQUE}

Nous appuyant sur différentes publications données en références (principalement [Grimal, 1993]), nous résumons comme repères historiques quelques dates des conquêtes et créations de provinces (cf. p. 43).

Il faut noter des datations parfois légèrement différentes selon les ouvrages traitant de l'Histoire romaine. Ceci s'explique par les incertitudes sur la durée de certaines guerres qui souvent se sont déroulées en plusieurs temps ou par les intervalles notables entre la fin des combats, les annexions et le passage des pays vaincus à celui de provinces romaines. Par exemple la province d'Asie n'a été créée qu'en -113 alors que la conquête s'était effectuée entre -133 et -129 . 


\begin{tabular}{|ll|}
\hline$-275 /-266$ & soumission de l'Italie du Sud \\
-241 & occupation de la Sicile \\
$-238 /-227$ & occupation de Corse et Sardaigne \\
-222 & occupation de la Gaule Cisalpine \\
-206 & création de la province Espagne \\
-146 & création de la province Afrique \\
-118 & création de la province de Gaule Narbonnaise \\
-113 & création de la province d'Asie \\
-100 & création de la province de Cilicie \\
$-74 /-69$ & création de la province de Crète et Cyrénaïque \\
$-74 / 62$ & création des provinces de Pont et Bithynie \\
-62 & création de la province Syrie \\
-51 & création de la province de la Gaule Chevelue \\
-46 & Africa Nova \\
-31 & Egypte et conquête du versant nord des Balkans \\
9 & abandon de l'Outre-Rhin (défaite de Varus en Westphalie) \\
42 & Maurétanie césarienne \\
43 & expédition de (Grande) Bretagne \\
$107-256$ & annexion de la Dacie \\
127 & mur d'Hadrien \\
273 & Fin de l'Empire des Gaules \\
364 & Byzance seconde capitale \\
\hline
\end{tabular}

Rappelons que ce sont les logarithmes de ces dates qui sont maniés dans ces calculs. On a donc affaire à un effet de nature statistique avec des pics de probabilités d'occurrence des événements. Une conséquence est que les petites imprécisions sur les dates n'ont guère une influence notable sur les résultats d'autant que les éléments du calcul ne sont pas les temps linéaires (c'est-à-dire les dates) mais des intervalles de temps.

La Figure 5 donne les principales conquêtes faites durant la République. Les flèches montrent bien la succession de celles-ci depuis Rome dans la direction des provinces espagnoles, vers différents points d'Afrique du Nord et surtout vers le MoyenOrient. Sont aussi représentées les annexions effectuées durant l'Empire, principalement par Trajan, pour consolider les frontières en appuyant celles-ci sur des obstacles naturels : l'Euphrate, le Rhin et le Danube, ou des côtes maritimes et le désert.

Dans l'histoire de la Rome Antique, schématiquement, on peut distinguer trois périodes. La première peut être considérée comme l'établissement du territoire avec l'annexion progressive des pays méditerranéens : Rome doit recouvrir le «Monde habité ». L'exemple à suivre est celui des conquêtes d'Alexandre le Grand. C'est l'époque républicaine qui va se terminer après la mort de César. Ces extensions ont amené à Rome des richesses (mines d'or et d'argent de Transylvanie, aliments d'Espagne, vins de Gaule, huile et blé d'Afrique du Nord et d'Egypte...). Auguste 
succède assez rapidement à César. Quelques échecs lui font comprendre qu'il faut ralentir puis cesser les annexions, ceci pour consolider l'Empire et le protéger. Ces objectifs sont assez largement poursuivis par ses successeurs. Comme les territoires romains sont très vastes (la quasi-totalité du Monde connu alors !), pour les sécuriser, presque toutes les légions sont regroupées en quelques points des frontières pour s'opposer aux «barbares » venus du Nord de l'Europe ou de l'Est. C'est alors que fut établi le «limes », frontière bien marquée par des constructions militaires (voies de circulation, casemates, murs...) dont l'exemple bien connu est le mur d'Hadrien en (Grande) Bretagne édifié quand le projet d'annexion de l'Ecosse fut abandonné. Enfin, à partir du dernier quart du IIe siècle, alors que certaines possessions romaines doivent être abandonnées, les Germains passent le Danube (le limes) et envahissent l'Italie (170180). Après 192, à la mort de Commode, c'est «la guerre des généraux », les commandants militaires. Malgré le rétablissement de l'Empire par Dioclétien, des tribus germaniques envahissent de nouveau celui-ci vers 270. C'est la partition entre l'Empire Oriental qui va résister jusqu'à l'occupation musulmane et l'Empire Occidental qui va rapidement se décomposer.

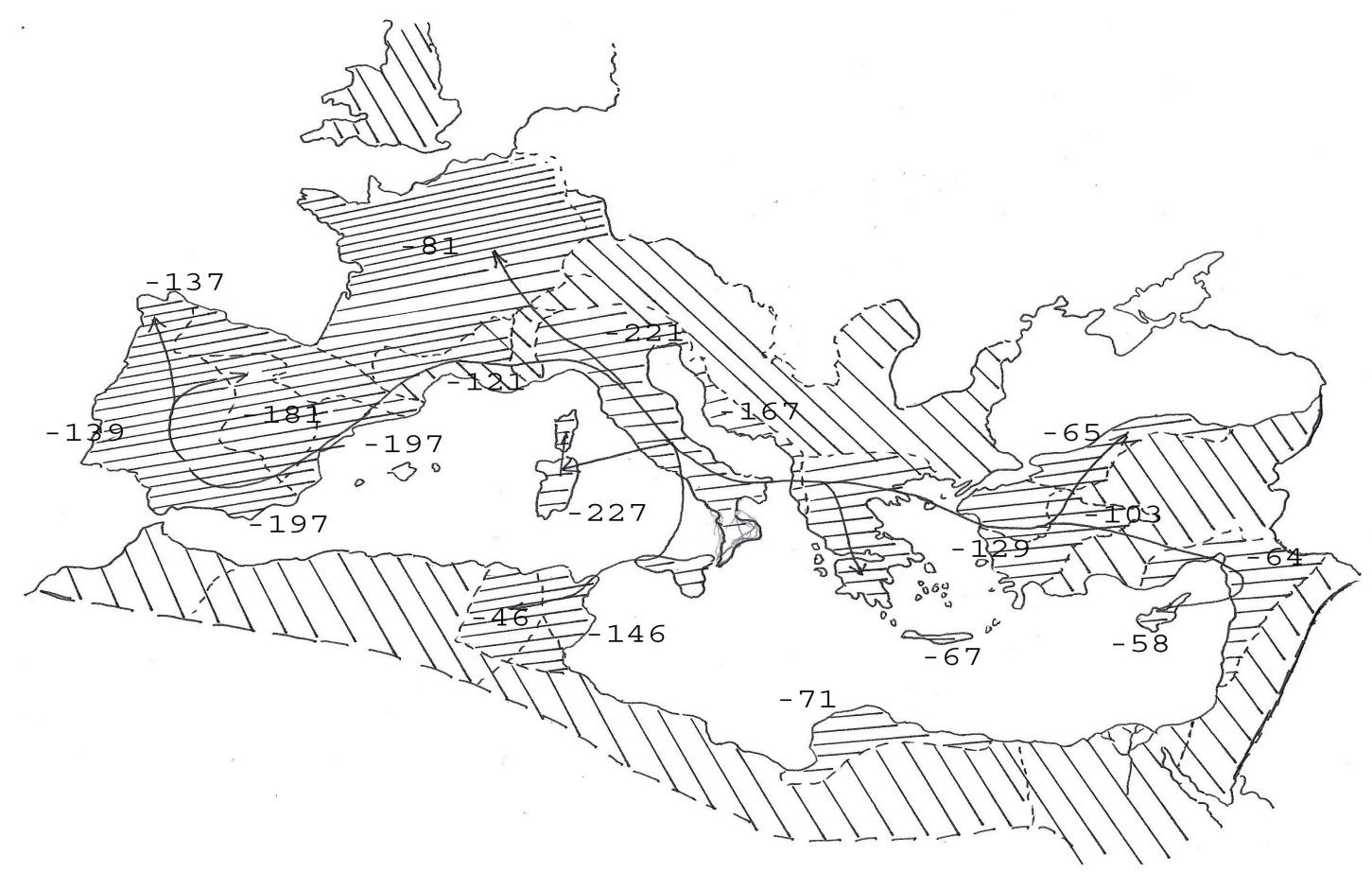

FIGURE 5. Annexions romaines durant la République avec la propagation de celles-ci (grisé foncé avec la date de la Conquête).

Le grisé clair correspond aux annexions durant l'Empire.

Exemple de carte utilisée pour évaluer les surfaces annexées

Dans un premier temps, nous nous intéresserons à la cartographie et à la date des conquêtes de la République romaine extraites des références. Il convient de bien préciser la technique opératoire : les valeurs des surfaces des territoires romains ont été obtenues par une série de «planimétrages» des cartes utilisées dont la Figure 5 donne un exemple. Ainsi chaque annexion est évaluée l'une après l'autre en prenant en compte la date qui lui est attribuée par l'historien concerné. Quant au Tableau 1, il a pour but de seulement rappeler des dates marquantes. Les valeurs des surfaces cumulées de ces 
conquêtes effectuées jusqu'à César, en fonction de leurs dates, sont présentées dans la Figure 6. La courbe correspondante peut être décomposée, grâce au logiciel Loglet Lab en trois courbes calculées avec trois fonctions logistiques de forme classique donnée plus haut. L'ajustement obtenu conduit à un accord très satisfaisant. On constate ainsi que les annexions se sont opérées en trois grandes vagues :

- la première vers -272 après la victoire sur Pyrrhus et le début de la conquête de l'Italie du Sud ;

- la suivante vers -195 avec l'annexion de la Grèce, avant l'annexion de différentes parties de l'Espagne après la défaite d'Hannibal (-183);

- enfin vers -85 c'est le début de l'absorption de plusieurs pays du Moyen Orient...

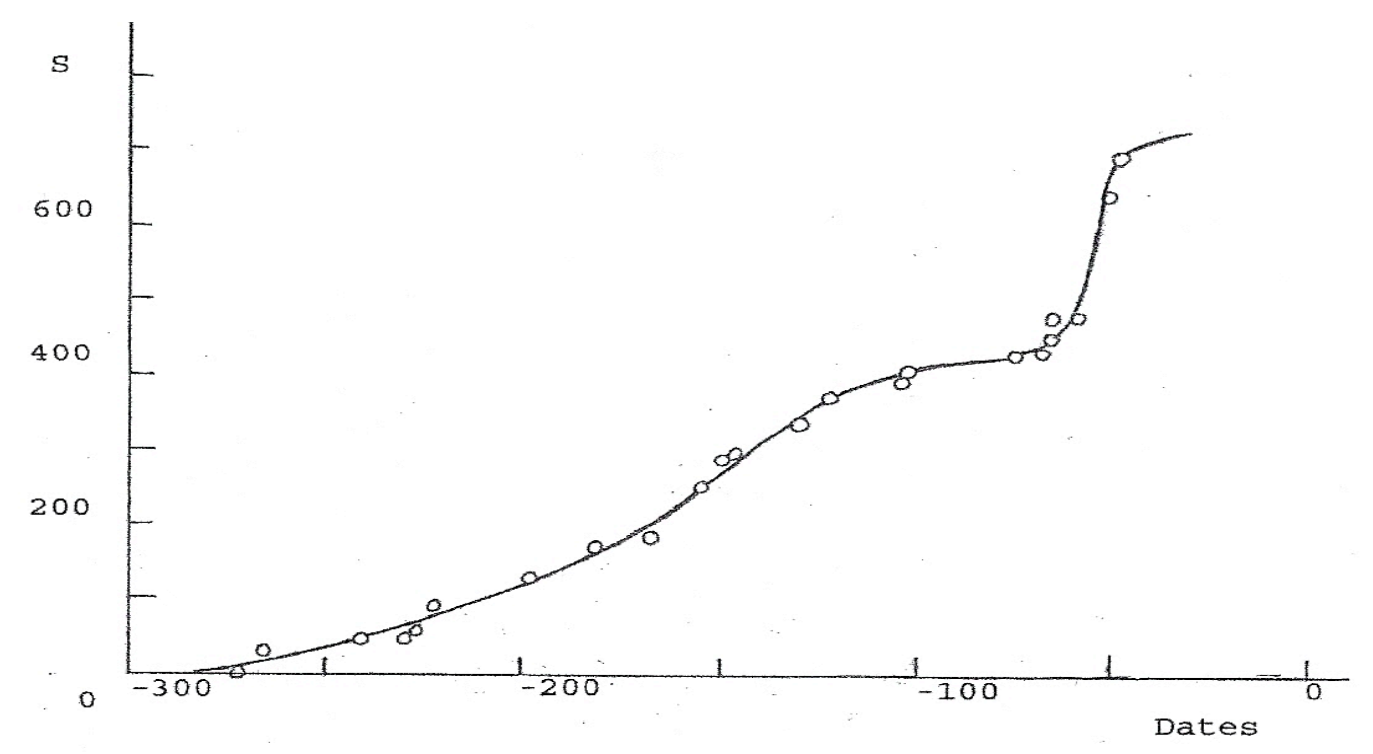

FIGURE 6. Surfaces cumulées des annexions romaines jusqu'à la mort de César

Comme le montre la carte de la référence [Chevallier, 1997] Rome au départ, une simple ville, a absorbé successivement les pays voisins avec des pauses nécessaires pour stabiliser ces nouvelles structures de leur puissance. Soulignons qu'en passant, ainsi, au cours de plusieurs siècles à ce grand ensemble de régions cerclant la Méditerranée, il y a eu une succession d'échelles dans la définition de Rome avec des déplacements du centre de gravité de l'État. La Figure 7 prolongeant la Figure 6, donne la surface cumulée des annexions après l'instauration de l'Empire par Auguste. Une seule fonction logistique peut très bien rendre compte de ces données. Soulignons au passage que l'ajustement de fonctions logistiques sur les données permet un lissage sur la courbe reliant celles-ci.

À l'aide des données précédentes on peut établir un arbre généalogique des conquêtes romaines. Celles-ci se sont réalisées par vagues, chacune de celles-ci toujours dans la même direction ; comme exemple, rappelons la conquête des différentes parties de l'Espagne. Bien que le nombre de ces vagues soit extrêmement faible, nous pouvons proposer qu'un modèle log-périodique puisse rendre compte de l'évolution de cette chronologie, dans le cas présent une décélération. 


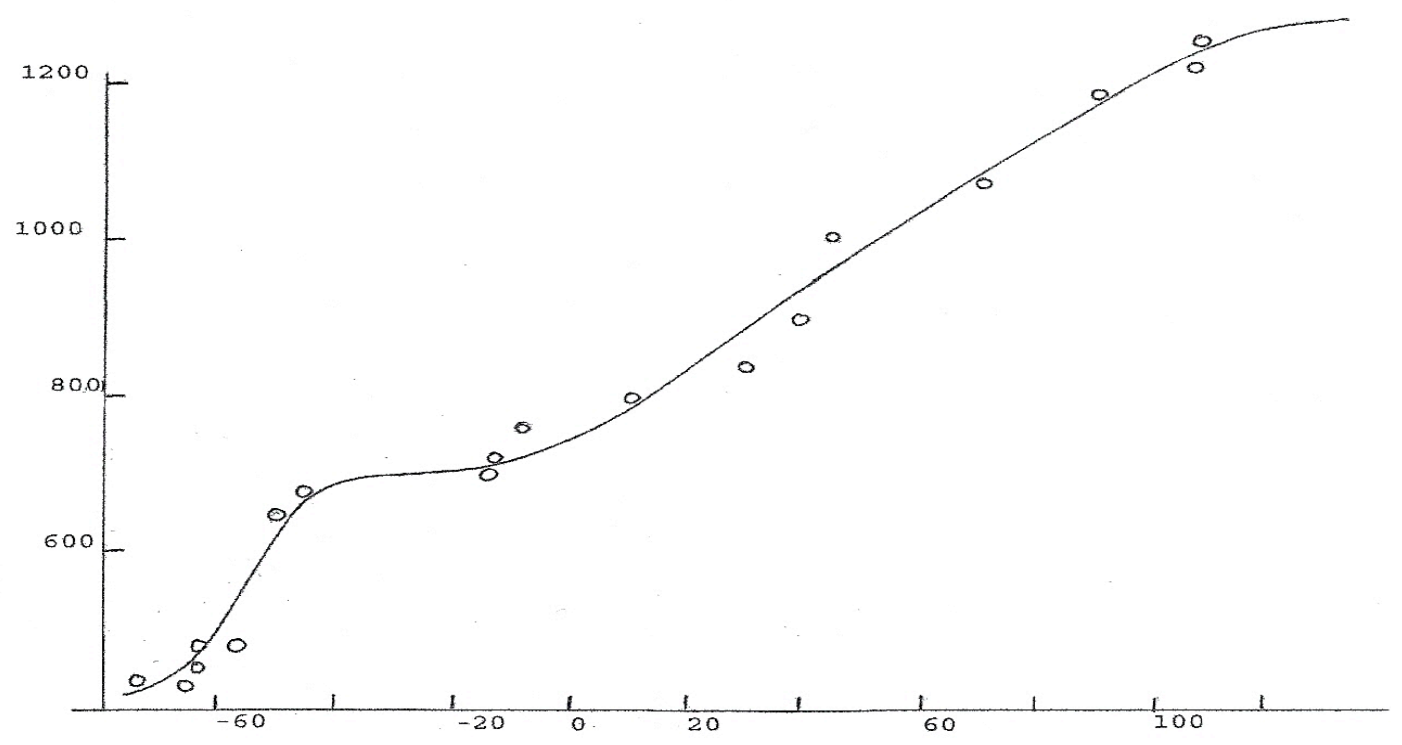

FIGURE 7. Surfaces cumulées par Rome jusqu'à Hadrien

Les équations de la log-périodicité (les relations I données plus haut) conduisent aux valeurs des deux ingrédients de la théorie :

\begin{tabular}{|lr|}
\hline coefficient d'autosimilarité & $g=1,30+/-0,15$ \\
temps critique & $T c=-490+/-30$ \\
\hline
\end{tabular}

Soulignons que cette valeur de $g$ concorde aux barres d'erreur près avec les valeurs de $g$ obtenues dans plusieurs autres analyses (pic de probabilité de $g$ pour $g 1=31 / 4=1.316$ ). D'autre part le temps critique trouvé ici $T c=-490+/-30$ est parfaitement en accord avec la date qu'avancent les historiens pour l'instauration de la République romaine à la place de la Royauté (509 av. J.-C.) quand Tarquin le Superbe est chassé et remplacé par deux consuls après une période de quelques années mouvementées.

Les équations de la log-périodicité prévoient comme date précédant celle de -272, la plage $-330+/-10$. Celle-ci tombe parfaitement dans l'intervalle de temps -336 à -327 qui a été nécessaire à Rome pour s'imposer dans le Sud de l'Italie et en Campanie, au départ des conquêtes. De même la date de nouvelles annexions calculée après l'établissement de l'Empire est +65 (après J.-C.) soit juste avant la conquête de l'Iran, l'Arabie... Ces dates sont en bon accord avec les résultats de la décomposition en fonctions logistiques.

Une conclusion de cette analyse est la constatation qu'après l'installation de l'Empire romain un processus de décélération du rythme des annexions s'est engagé avant d'être stoppées. Un parallèle est possible avec le rythme des répliques suivant un séisme et s'atténuant peu à peu avant d'être imperceptibles. Comme les annexions étaient nécessaires pour la protection, l'entretien, la survie de l'Empire, on peut comprendre que celui-ci se soit assez rapidement fragilisé quand le rythme des annexions s'est réduit. 


\subsection{LES VOIES ROMAINES}

Les routes construites uniquement en Italie ont été bien étudiées et parfaitement datées. Sur la Figure 8 sont portées les nombres cumulés de ces voies en fonction de la date de construction. Déjà, on voit la confirmation qu'elles ont été réalisées bien avant les annexions successives des régions de la péninsule précisées plus haut : par exemple, la première (la Via Appia) date de 312 av. J.-C. Ultérieurement, cet équipement routier n'évolue guère en Italie quand l'Empire succède à la République. Par contre l'Empire devra augmenter le réseau routier dans toutes les autres provinces pour structurer la défense de son territoire qui, alors, a presque atteint sa surface maximum.

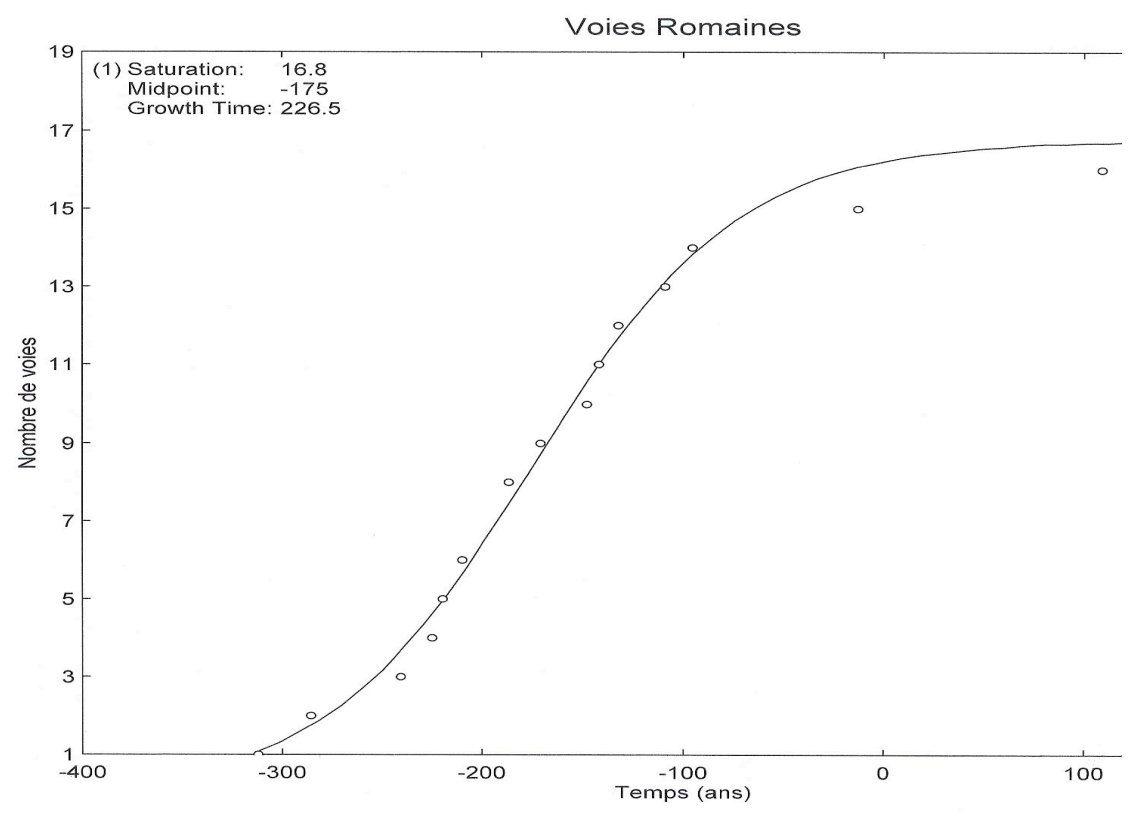

FIGURE 8. Nombre cumulé des voies romaines en Italie.

La courbe théorique est une logistique.

La courbe théorique portée sur la Figure 8 correspond à une fonction logistique qui rend bien compte de la diffusion de cette innovation technique qu'est la réalisation d'une voie de circulation. L'accord avec les données est excellent. Il n'y a plus de voie importante réalisée à partir du premier siècle après $\mathrm{J}$.-C. exceptée dans cette région. Il est évident que dans les autres régions de l'Empire on doit constater la même forme logistique de diffusion.

Le tableau suivant donne la liste des voies romaines que l'on peut considérer comme point de départ d'une série de construction dans une direction donnée, par exemple vers l'Espagne, vers la Sicile, vers la Grèce, etc... ; ce sont des repères dans la chronologie du réseau routier : 


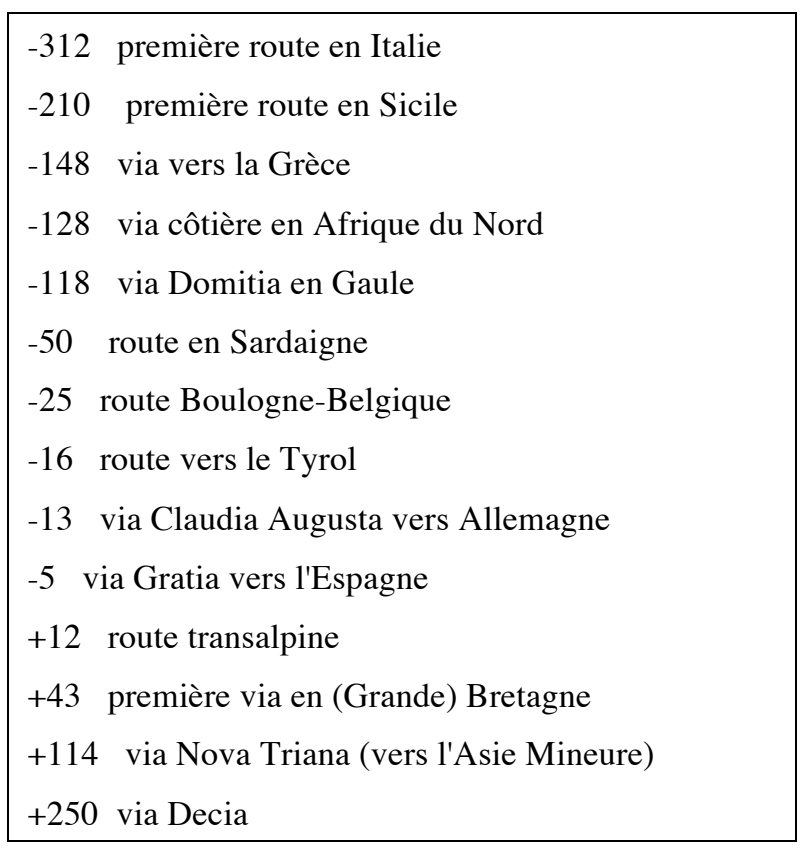

Un coup d'œil montre qu'il y a dans cette chronologie une accélération du rythme des constructions jusqu'au premier siècle avant J.-C. suivie d'une décélération. L'ajustement (grâce aux équations I) d'une loi log-périodique successivement sur ces deux périodes aboutit pour la période républicaine aux deux paramètres suivants :

$$
g=2,20+/-0,40 \quad \text { et } \quad T c=110 \text { av. J.-C. }
$$

et pour la période impériale:

$$
g=2,15+/-0,30 \quad \text { et } \quad T c=17 \text { apr. J.-C. }
$$

Notons la compatibilité aux incertitudes près des deux valeurs de $g$ entre elles et avec $g 3=33 / 4=2.28$ qui est un des pics de probabilité observé pour les valeurs de $g$ obtenues dans diverses sciences [Nottale, 2010].

Sur la Figure 9 la courbe donne le nombre approximatif cumulé de routes romaines (du moins de celles dont nous avons pu avoir la date) en fonction de la date de réalisation. Comme précédemment, on distingue deux parties : la première correspond à la période républicaine durant laquelle furent construites les routes permettant les annexions des pays bordant la Méditerranée. Cette politique s'arrête au milieu du premier siècle avant J.C. La seconde partie montre, avec des échelles différentes, la construction des nombreuses routes durant l'Empire dans le cadre du retranchement, quand il a été nécessaire de renforcer les défenses des régions annexées. Mais au-delà de 250 après J.-C. le nombre de voies n'évolue que peu.

\subsection{DISCUSSION ET CONCLUSION}

Nous constatons que la courbe 8 et la courbe 6 donnant la surface cumulée des annexions sont très voisines. Ceci confirme quantitativement la corrélation entre les conquêtes et la construction des voies malgré des buts a priori assez différents. Dans les deux cas il faut noter un ralentissement des deux processus à la fin du dernier siècle avant J.-C. Également, au cours du IIe siècle après J.-C., les annexions se font rares (les Romains abandonnent même des territoires trop difficiles à conserver) et la construction des routes s'arrête. C'est le début du déclin. 


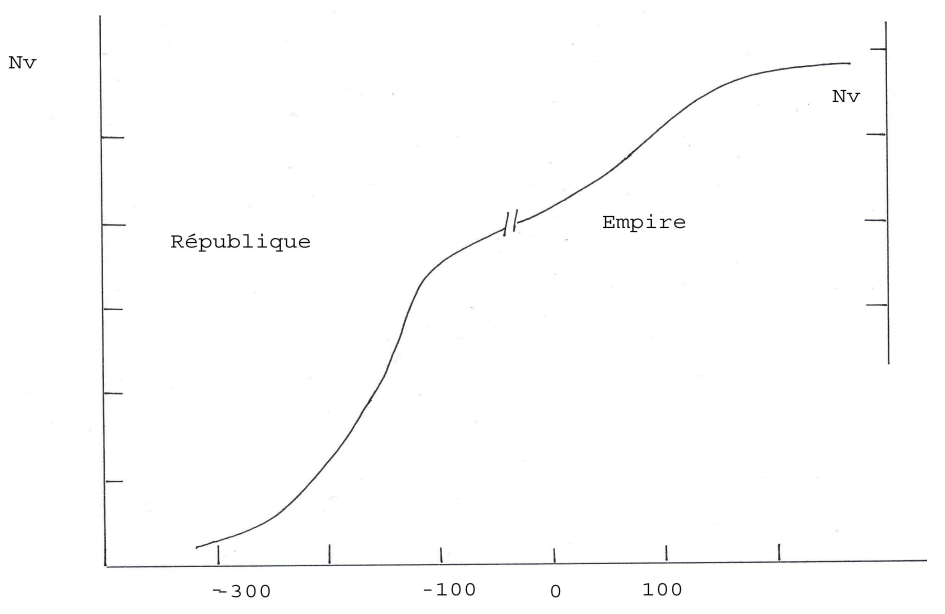

FIGURE 9. Courbes donnant le nombre cumulé de voies romaines pendant la République et l'Empire.

Les échelles sont différentes pour ces deux courbes

L'Empire romain a été brillant avec des innovations et des succès dans de nombreux domaines. Sa prééminence avait succédé à celle de la Grèce quand cette dernière n'a plus eu les moyens de dominer le monde méditerranéen. Rome a eu le même destin face à Byzance. Après une période prospère les crises n'ont plus permis de s'opposer à la puissance adverse. Dans cet article nous pouvons constater que le déclin de Rome suit un cheminement voisin de celui qu'a parcouru la Russie ultérieurement, cheminement qui s'explique bien en termes d'accélérations et de décélérations logpériodiques.

Nous avons montré que les Empires de Russie et de Rome ont évolués sur des rythmes log-périodiques. Évidemment il reste à vérifier que d'autres empires ont évolué également en suivant une même loi. Mais l'hypothèse du travail est celle d'une universalité pour de nombreux phénomènes évolutifs et que cette chronologie est multi échelle. Nous l'avons montré [Nottale, Chaline, Grou, 2000] pour l'évolution globale entre les empires. Le présent travail correspond à l'échelle inférieure (à l'intérieur d'un empire donné) pour deux d'entre eux. Nous avons l'intention de poursuivre cette étude sur d'autres empires pour lesquels suffisamment de données sont disponibles.

Il faut noter la différence importante entre les valeurs de $g$ obtenues dans l'analyse des conquêtes et celles déduites de l'analyse des constructions de voies. Dans ce dernier cas les coefficients d'autosimilarité sont bien supérieurs ; ils signifient, sans doute, que les étapes sont plus vite franchies. Une explication peut être avancée : les techniques de construction des voies sont bien maîtrisées par les Romains même dans des régions délicates. Par contre, les conditions pour mener à bien leurs conquêtes, par des moyens militaires, diplomatiques ou les deux, sont toujours fort différentes, d'où des étapes parcourues sur un rythme plus lent. Nous sommes arrivés à la même conclusion dans des cas divers [Brissaud, 2009]. Il est aussi particulièrement intéressant de constater que les coefficients $g$ sont très voisins dans l'évolution des conquêtes russes et romaines, alors que ces évolutions se sont effectuées dans des régions et à des époques fort dissemblables. Il nous faut donc comprendre cette universalité (les coefficients obtenus dans cette étude, $1.30 \pm 0.15,1.71 \pm 0.04$ et $2.2 \pm 0.2$ sont tous compatibles avec une loi en $3 k / 4$, avec $k=1$ à 3 ).

Mais, si la surface du territoire romain ne croît plus, si la réalisation des voies cesse vers la fin de l'Empire, le tempo de l'évolution n'est pas identique : les conquêtes 
se sont effectuées tout au long des quatre à cinq siècles pris en compte ici avec, certes un rythme plus grand avant J.-C. alors que des voies romaines ont été rapidement établies lors du développement de la République jusqu'à la mort de César. Après une pause relative à l'époque d'Auguste la construction des routes a repris de plus belle quand la politique d'accroissement des défenses s'est imposée.

Dans cette analyse log-périodique du développement de la Rome antique le facteur d'autosimilarité a la valeur $g=1,30+/-0,15$ nettement inférieure à la valeur obtenue dans l'analyse de l'expansion russe $g=1,71+/-0,04$. Remarquons que l'on obtient une valeur $g=1,32+/-0,02$ dans l'étude de la succession des crises économiques [Grou, Nottale, Chaline, 2002] et que $g=2,3+/-0,2$ dans l'analyse de la succession des crises économiques de l'URSS [Nottale, Chaline, Grou, 2009]. Les coefficients obtenus dans la présente étude, et dans ces études précédentes sont tous compatibles avec une loi en $3 k / 4$, avec $k=1$ à 3 . Ce facteur $g$ peut être compris comme l'indication de la vitesse de passage d'une poussée d'annexions à la suivante. Plus $g$ est grand et plus cette vitesse est grande. Il a été suggéré qu'un effet de mémoire gère cette vitesse : mémoire permettant de gagner du temps pour passer à l'étape suivante. Pour la Russie, la persistance quasi-continue de conflits (comme le prouve l'histoire du pays) a maintenu un état de tension quasi-permanent ; cela a-t-il facilité les occupations territoriales ? Nottale [1998] a avancé une explication : l'optimisation sous contrainte qu'il définit ainsi :

$$
\begin{aligned}
& \text {... après une première dilatation générique qui a permis l'optimisation, il y a, } \\
& \text { à nouveau, blocage du système, qui se trouve ramené à l'état précédent, au } \\
& \text { facteur d'échelle près. Le problème étant le même, la solution est également } \\
& \text { semblable, si bien qu'une nouvelle itération peut opérer. }
\end{aligned}
$$

Enfin nous avons souligné que l'on a rencontré dans ces deux analyses plusieurs caractéristiques déjà notées par NCG : présence de la complexité, apparition de plusieurs échelles dans l'évolution des surfaces territoriales et du nombre de voies, recherche de gains économiques plus aisés dans ces évolutions, accélération et décélération de celles-ci et prédictibilité. Tout ceci nous a donc confortés dans l'application de la TRE. Or, un des buts de ce travail est de vérifier que la TRE s'applique à un grand nombre de phénomènes dans des domaines variés afin de conforter la validité de la théorie de Nottale. Par ailleurs la présence d'un processus logpériodique peut être profitable pour les historiens.

Après avoir établi une chronologie log-périodique les historiens peuvent détecter un « vide » dans la succession et en déduire un « saut» non encore identifié jusqu'alors. De telles déductions ont été faites dans diverses analyses de NCG.

La TRE peut aussi être féconde pour les historiens en permettant de prévoir avec une certaine précision statistique les dates des événements à venir (seulement les dates). Il suffit de disposer de trois événements bien établis pour calculer les dates des événements suivants. Mais plus on a à sa disposition un grand nombre de dates, meilleure est la précision sur les prévisions.

Il est également intéressant pour les historiens de constater (comme ici) l'action d'un hasard contraint et non la présence du hasard bénin pour piloter des évolutions. Il faut bien préciser que la log-périodicité prévoit qu'une évolution étudiée se dirige vers un instant critique avec une incertitude sur la date de celui-ci, mais surtout avec l'ignorance des événements qui provoqueront la transition au temps $T c$ et aussi les conséquences de celle-ci. L'analyse de ces événements critiques et de ses conséquences reste du domaine des historiens. Des collaborations avec ces derniers semblent donc nécessaires et prometteuses. 
Dans cette étude de la «longue durée » a été menée en ne prenant en compte que l'espace et le temps. Mais ceci n'est pas exclusif. Il est possible de la reprendre avec d'autres paramètres dans des études à venir. Rappelons que nous nous sommes appuyés dans cette dernière analyse sur les surfaces des annexions et également sur les nombres de voies romaines. Cependant, nous avons bien distingué les buts à long terme atteints grâce à la TRE alors que certaines caractéristiques des pays étudiés permettent de comprendre leur évolution sur des temps plus courts avec, certes, plus de sûreté et de précision.

Remerciements. Nous tenons à remercier nos collègues historiens Serge Chassagne (Université Lyon 2) pour avoir relu avec beaucoup d'attention ce texte et Jean-Pierre Chaline (Université Paris-Sorbonne) pour de fructueux et passionnés commentaires. Christiane Boghossian (MSH) nous a apporté une aide précieuse dans la mise au point du texte. Nous tenons à lui exprimer toute notre gratitude.

\section{BIBLIOGRAPHIE}

BORDET M. (2004), Précis d'histoire romaine, Paris, Armand Colin.

BRISSAUD I. (2007), «La chronologie du jazz suit-elle une loi log périodique ? », Mathématiques et Sciences humaines 178 , p. 41-50.

BRISSAUD I., BARON E. (2007), « La Course des accélérateurs de particules vers les hautes énergies et la log-périodicité », Revue Européenne de Géographie, Cybergeo ; http://www.cybergeo.eu/index 14173.html.

BRISSAUD I. (2009(a)), «Évolution log-périodique des ordres religieux et monastiques », Mathématiques et Sciences Humaines, p. 55-65.

BRISSAUD I. (2009(b)), «Un exemple de log-périodicité: la découverte des éléments chimiques », Compterendu de l'Académie des Sciences (Chimie) 12, p. 1313-1318.

BRONINA E., JOUKOV V. (1962), Histoire de l'URSS, Moscou, éditions en langues étrangères.

CHALINE J., NOTTALE L., GROU P. (1999), «L'arbre de vie a-t-il une structure fractale ? », Comptes Rendus de l'Académie des Sciences 328, p. 717-726.

CHANNON J. (1997), Atlas historique de la Russie, Paris, éditions Autrement.

CHEVALIER R. (1997), Les voies romaines, Paris, Picard.

COULON G. (2009), Les voies romaines en Gaule, Paris, éditions Errance.

DUPUY J. (dir.) (1999), Histoire de la Rome antique, Trois continents, Paris, édition du Rocher.

GRIMAL P. (1993), L'Empire Romain, Paris, éditions Fallois.

GROU P. (1987, 1995), L'Aventure économique, Paris, L'Harmattan.

GROU P., NOTTALE L., CHALINE J. (2002), "Log-periodic laws applied to geosciences. Miscelanea en homenaje a E. Aguirre", Zona Arquelogica, Arqueologia 4, p. 230-237.

GROU P. (2010), L'argent, obscurantisme du XXIe siècle, Paris, Bruno Leprince.

HERMANN P. (2007), Itinéraires des voies romaines de l'Antiquité au Moyen-Âge, Paris, éditions Errance.

JOHANSEN A., SORNETTE D. (2001), "Finite-time singularity in the dynamics of the world population and economic indices”, Physica A 294, p. 465-502.

NOTTALE L. (1993), "Fractal Space and Microphysics. Towards a Theory of Scale Relativity", Singapore, World Scientific.

NOTTALE L. (1998), La relativité dans tous ses états, Paris, Hachette.

NOTTALE L., CHALINE J., GROU P. (2000(a)), "On the fractal structure of evolutionary trees", Fractales en biologie et en médecine, vol. III, Actes du IIIe International Symposium, Ascona (Suisse), 8-11 mars 2000, G. Losa, D. Merlini, T.F. Nonnenmacher, E.R. Weibel (eds), Birkhäuser Verlag, p. 247-258.

NOTTALE L., CHALINE J., GROU P. (2000(b)), Les arbres de l'évolution, Paris, Hachette. 
NOTTAle L., CHALINE J., GROU P. (2009), Des fleurs pour Schrödinger. La Relativité d'échelle et ses applications, Paris, Ellipses.

PRECHAC A. (2001), Histoire de la Russie avant 1917, Paris, Bordas.

RADVANYI J. (2004), La nouvelle Russie, Paris, Armand Colin.

SCARRE C. (1995), Atlas de la Rome Antique, Paris, éditions Autrement.

SORNETTE D., SAMMIS C.G. (1995), "Complex critical exponents from renormalization group theory of earthquakes", Journal de Physique I (5), p. 607-619.

SORNETTE D., JOHANSEN A., BOUCHAUD J.-P (1996), "Stock market crashes, precursors and replicas", Journal de Physique I(6), p. 167-175.

SORNETTE D. (2002), Why stock markets crash, Princeton, Princeton University Press. 